\title{
Preliminary results on the study of the environment of a complete sample of Seyfert galaxies *
}

\author{
M. Salvato and P. Rafanelli, Padova, Italy \\ Department of Astronomy, University of Padova
}

Received 1997 May 24; accepted 1997 June 3

\begin{abstract}
The results of the study of the environment of a complete sample of Seyfert galaxies taken from the CfA Redshift Survey (Davis et al. 1983; Huchra et al. 1983) are shown. In particular we compare the distribution of the positions of all galaxies located within five diameters from each Seyfert galaxy of our sample with the analogous distribution observed in a control sample of normal galaxies taken from the Merged Catalogue of Galaxies (hereafter MERCG) (Kogoshvili 1986). This research is based on the analysis of the digitized images of the "Digitized Sky Survey" and on the on-line catalogues APM (Automatic Plate Measuring System) and APS (Automated Plate Scanner).
\end{abstract}

Key words: active galaxies: Seyfert galaxies

AAA subject classification: 158

1.

\section{Introduction}

The most popular mechanism to explain activity in galaxies is accretion of gas onto a massive black hole (Gunn 1979). N-body simulations (for example those of Barnes \& Hernquist 1991) demonstrate how tidal forces produced by interaction with companions of comparable or much smaller mass might contribute to solve the problem of gas transport into the nuclear regions of a galaxy.

Statistical studies aimed to demonstrate an excess of interacting systems among Seyfert galaxies, gave until recently conflicting results. According to Dahari (1985) and MacKenty (1989) there is an excess among Seyfert galaxies of galaxies with nearby companions. Fuentes-Williams and Stocke (1988) achieved an opposite result. Laurikainen \& Salo (1995) found that type 1 and type 2 Seyfert galaxies (hereafter Sy1 and Sy2, respectively) have different environments; the frequency of Sy2 in interacting systems is larger than that of Sy1, which appear in interacting systems as often or even less frequently than the control galaxies. This result is in disagreement with the idea of an unified model for Seyfert galaxies. The main reason for these conflicting results is quite likely in the selection criteria used to define the samples of Seyfert and control galaxies. The only study in which the active galaxies of the sample have been extracted from lists of Seyfert galaxies, identified in spectroscopic surveys, namely independently from their morphology, is that of Rafanelli et al. (1995) who found an excess of paired systems among Sy1 and Sy2 compared to a control sample of normal galaxies. The lower limit for the percentage of physical companions is $12 \pm 3 \%$ for Sy 1 and $12 \pm 4 \%$ for Sy 2 , a clear excess respect to $\sim 0-5 \%$ found in the control sample.

Until now more attention has been paid to the search for companions of Seyfert galaxies than to the analysis of the environment in which they are embedded. This is the aim of the present work. In Sect. 2 we show how the samples have been defined. In Sect. 3 we present the data and the results.

2.

The sample of Seyfert galaxies and the control sample

The sample of active galaxies has been extracted from the complete sample of Seyfert galaxies identified in the CfA Redshift Survey by Huchra \& Burg (1992). It contains 25 galaxies (13 Sy1 and 12 Sy2) in the magnitude range $14 \leq m_{b} \leq 14.5$ where $m_{b}$ is the magnitude in the uncorrected $\mathrm{B}(0)$-Zwicky system (Huchra 1976). The objects of

\footnotetext{
*Poster presented at the Asiago Meeting "Environmental and physical properties of nearby AGN" on 23-24 April, 1997
} 
our sample are listed in Table 1 (column [1]) together with their coordinates (cols. [2] and [3]), the magnitude $m_{b}$ (col. [4]), the heliocentric velocity in km/s (col. [5]) and the Seyfert type (col. [6]), as given by Huchra \& Burg. Column [7] gives the density of galaxies per square arcmin calculated over a circular area of sky centered on the galaxy being studied and with a radius 5 times its major axis.

Table. 1. Sample of Seyfert galaxies

\begin{tabular}{|l|c|c|c|c|c||c|}
\hline $\begin{array}{c}\text { Name } \\
(1)\end{array}$ & $\begin{array}{c}\text { R.A. (1950) } \\
(2)\end{array}$ & $\begin{array}{c}\text { Decl. (1950) } \\
(3)\end{array}$ & $\begin{array}{c}m_{b} \\
(4)\end{array}$ & $\begin{array}{c}V_{H} \\
(5)\end{array}$ & $\begin{array}{c}\text { Type } \\
(6)\end{array}$ & $\begin{array}{c}\rho \\
(7)\end{array}$ \\
\hline Mrk 334 & $00^{h} 00^{m} 35^{s} .6$ & $21^{\circ} 40^{\prime} 54^{\prime \prime}$ & 14.40 & 6605 & 2 & 0.20 \\
Mrk 335 & 000345.2 & 195529 & 14.00 & 7757 & 1 & 0.27 \\
A0048+29 & 004853.1 & 290746 & 14.50 & 10763 & 1 & 0.26 \\
IZW 1 & 005057.9 & 122518 & 14.30 & 18116 & 1 & 0.12 \\
Mrk 993 & 012242.7 & 315236 & 14.00 & 4625 & 2 & 0.31 \\
Mrk 573 & 014122.9 & 020557 & 14.00 & 5178 & 2 & 0.19 \\
0152+06 & 015244.7 & 062202 & 14.50 & 5208 & 2 & 0.15 \\
NGC 863 & 021200.5 & -05958 & 14.00 & 7891 & 1 & 0.22 \\
NGC 3080 & 095714.2 & 131703 & 14.50 & 10602 & 1 & 0.20 \\
A1058+45 & 105842.5 & 455522 & 14.10 & 8778 & 2 & 0.34 \\
Mrk 205 & 121933.5 & 753610 & 14.50 & 20978 & 1 & 0.25 \\
Mrk 231 & 125405.4 & 570840 & 14.10 & 12287 & 1 & 0.08 \\
1335+39 & 133528.4 & 392431 & 14.20 & 6023 & 2 & 0.33 \\
NGC 5252 & 133544.4 & 044747 & 14.50 & 6926 & 2 & 0.18 \\
NGC 5256 & 133614.7 & 483153 & 14.10 & 8239 & 2 & 0.19 \\
NGC 5283 & 133941.2 & 675536 & 14.30 & 2697 & 2 & 0.15 \\
Mrk 461 & 134504.4 & 342357 & 14.50 & 4894 & 2 & 0.17 \\
Mrk 279 & 135152.5 & 693316 & 14.50 & 9129 & 1 & 0.21 \\
Mrk 817 & 143457.9 & 590039 & 14.30 & 9430 & 1 & 0.45 \\
Mrk 841 & 150136.3 & 103756 & 14.00 & 10921 & 1 & 0.37 \\
NGC 5929 & 152418.9 & 415041 & 14.00 & 2504 & 2 & 0.16 \\
NGC 5940 & 152851.3 & 073738 & 14.30 & 10160 & 1 & 0.31 \\
NGC 6104 & 161440.1 & 354950 & 14.10 & 8397 & 1 & 0.18 \\
Mrk 530 & 231622.6 & -00139 & 14.40 & 8691 & 1 & 0.18 \\
NGC 7682 & 232630.7 & 031528 & 14.30 & 5107 & 2 & 0.13 \\
\hline
\end{tabular}

The control sample has been built taking from the MERCG for each Seyfert galaxy, the nearest normal spiral galaxy having an angular diameter $D_{N}$ :

$0.9 D_{S y} \leq D_{N} \leq 1.1 D_{S y}$

where $D_{S y}$ is the angular diameter of the associated Seyfert galaxy. The data of these galaxies are reported in Table 2.

It is worthwhile to note that the distribution of the diameters of the galaxies of both samples (Fig. 1) arise from the same generating function at a confidence level of $98.8 \%$ as derived from a Kolmogorof-Smirnov test.

3.

Data and results

The environment of each galaxy of the two samples has been investigated on the red images of the Digitized Sky Survey. Firstly we have measured the angular size in arcmin of the major $\left(D_{i}\right)$ and minor $\left(d_{i}\right)$ axes of the galaxies of our samples, where $i=1,2, \ldots 25$ is the sequence number of the galaxies in Tab.1 and Tab.2. Then we have identified around each of them all galaxies located within a circular area of radius equal to five times the size of the major axis $D_{i}$ using APM. For each new identified galaxy it has been measured:

- the position,

- the distance from the central galaxy,

- the size of the major and minor axes,

- position angle of the major axis,

- the $\mathrm{R}$ magnitude. 


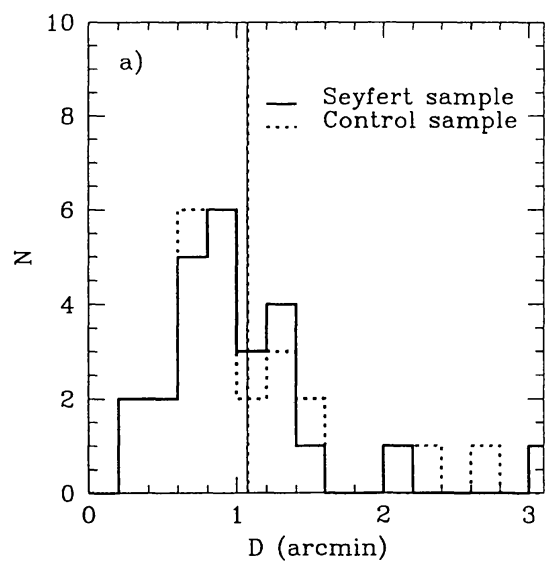

Fig. 1. Distributions of diameters of the Seyfert galaxies (solid line) and of the galaxies of the control sample (dotted line). The vertical lines show the average values for both samples.

Table. 2. Galaxies of the control sample

\begin{tabular}{|c|c|c|c|c|c|}
\hline $\begin{array}{c}\text { Name } \\
(1)\end{array}$ & $\begin{array}{l}\text { R.A. }(1950) \\
(2)\end{array}$ & $\begin{array}{c}\text { Decl. (1950) } \\
\text { (3) }\end{array}$ & $\begin{array}{l}m_{b} \\
(4)\end{array}$ & $\begin{array}{l}V_{H} \\
(5)\end{array}$ & $\begin{array}{c}p \\
(6)\end{array}$ \\
\hline $\mathrm{MCG}+05-01-000$ & $23^{h} 56^{m} 46^{s} .90$ & $31 \circ 00^{\prime} 24.0^{\prime \prime}$ & 16.0 & 5004 & 0.09 \\
\hline MCG+01-01-000 & 235859.80 & 084402.0 & 15.18 & 9255 & 0.15 \\
\hline MCG+05-01-007 & 235333.24 & 290558.7 & 15.45 & 6939 & 0.07 \\
\hline $\mathrm{MCG}+01-03-000$ & 005425.65 & 070333.0 & 16.5 & & 0.11 \\
\hline MCG+05-03-024 & 005224.60 & 311615.0 & 13.39 & 5460 & 0.23 \\
\hline MCG +03-04-036 & $\begin{array}{lll}01 & 23 & 15.50\end{array}$ & 162033.0 & 14.19 & 4134 & 0.32 \\
\hline MCG +03-04-038 & 012403.00 & 170020.0 & 14.81 & 2592 & 0.09 \\
\hline MCG+02-05-035 & 014554.00 & 101923.0 & 16.0 & 5271 & 0.07 \\
\hline MCG $+04-22-000$ & 092302.90 & 242120.0 & 15.7 & & 0.22 \\
\hline MCG+09-16-051 & 094433.50 & 541450.0 & 14.06 & 7407 & 0.31 \\
\hline$M C G+11-15-064$ & $1232 \quad 20.22$ & 634810.1 & 12.99 & 2733 & 0.12 \\
\hline$M C G+12-12-022$ & $1255 \quad 29.15$ & 702834.4 & 14.61 & & 0.21 \\
\hline $\mathrm{MCG}+09-22-030$ & 131113.83 & 514331.7 & 16.5 & & 0.04 \\
\hline MCG+03-34-005 & 131106.67 & 161522.2 & 14.96 & 6798 & 0.16 \\
\hline MCG+09-23-019 & $1355 \quad 17.16$ & 504045.2 & 16.0 & & 0.11 \\
\hline MCG+13-09-003 & 114634.40 & 743449.0 & 14.15 & 6819 & 0.10 \\
\hline MCG+06-32-000 & 142600.00 & 340232.1 & 16.0 & 4095 & 0.12 \\
\hline MCG +13-10-000 & 133127.46 & 770549.3 & 15.5 & & 0.13 \\
\hline$M C G+10-21-043$ & 145956.96 & 602624.0 & 16.0 & & 0.15 \\
\hline$M C G+04-34-050$ & 143530.57 & 213428.4 & 15.00 & 5427 & 0.12 \\
\hline MCG+09-24-011 & 143128.79 & 531155.0 & 16.0 & & 0.12 \\
\hline MCG+03-38-085 & 150306.48 & 143718.0 & 15.02 & & 0.21 \\
\hline MCG+08-29-049 & 160825.89 & 491745.7 & 16.0 & & 0.13 \\
\hline$M C G+02-01-002$ & 235424.40 & 103248.0 & 14.91 & 5214 & 0.19 \\
\hline MCG+03-60-006 & 233222.30 & 170114.0 & 15.62 & 6726 & 0.08 \\
\hline
\end{tabular}

Since the magnitudes given by APM are reliable for $m>18$ and those given by APS are reliable for $m<20$ we have used both catalogues for their determination (Salvato 1996). Here we will use only the position of the galaxies relative to the central object. The other parameters will be used in a future more detailed work.

The densities of galaxies per square arcminute, $\rho_{i}=N_{i} / A_{i}$ (where $N_{i}(1 \leq i \leq 25)$ is the number of galaxies in a circular area $A_{i}=\pi\left(5 D_{i}\right)^{2}$ centered on the $i$-th galaxy with major axis $\left.\overline{D_{i}}\right)$ are given in Tables 1 and 2 . The mean density of galaxies around the Seyfert galaxies is $30 \%$ larger than around the non active galaxies of the control sample.

In order to analyze the distribution of density of galaxies around Seyfert and normal galaxies we have taken for each galaxy as unity of length its angular diameter $D_{i}$. The galaxies surveyed in the 25 Seyfert environments ( 800 objects) have been then collected together around a fictitious galaxy with major axis D. The same was done for the galaxies surveyed in non active environments (516 objects) (Fig. 2 a) and b)). This operation is allowed by the good agreement found in the distribution of diameters of the galaxies of the two samples.

in a further step we have measured the number of galaxies in circular rings of thickness $D$ and area 

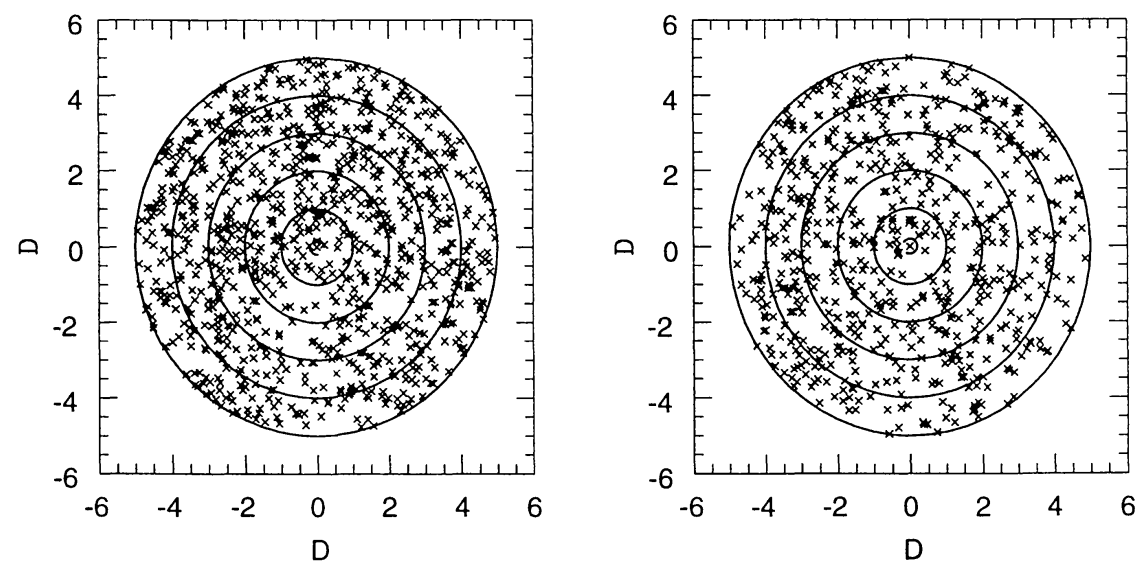

Fig. 2. Position of the galaxies identified in the two samples of Seyfert (left) and normal (right) galaxies.

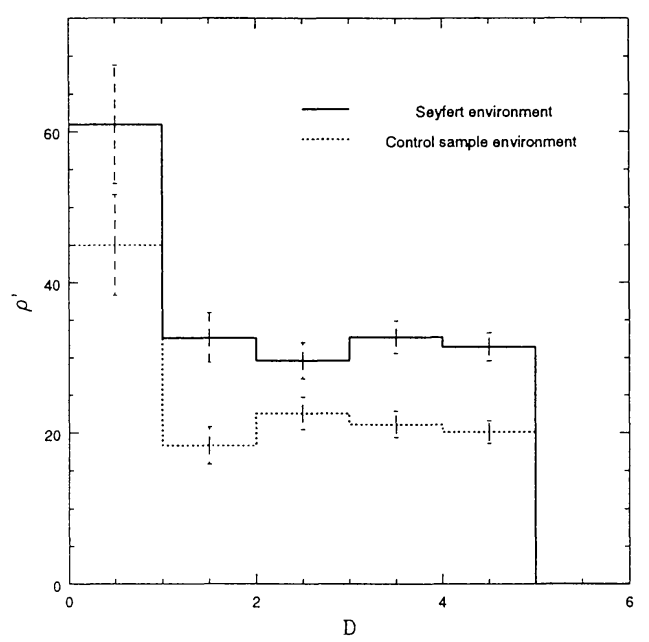

Fig. 3. In the histogram are shown the values of $\rho^{\prime}$ in Seyfert environment ( solid line) and non active environment (dotted line).

$$
S_{j}=(2 j-1) \pi D^{2}(j=1,2, . .5),
$$

The number of galaxies in each ring has been divided by $K_{j}=2 j-1$ and the resulting densities $\rho^{\prime}=N_{j} / K_{j}$ (densities which use as area unit $\pi D^{2}$ ) are plotted in a histogram in Fig. 3. as a function of $D$. The error associated to each value of $\rho^{\prime}$ is

$$
\left.\sigma=\sqrt{(} N_{j}\right) / K_{j} .
$$

It is remarkable to note that the histrogram shows a systematic constant excess of galaxies in the Seyfert environment (solid line) compared to the number of galaxies in the non active environment (dashed line).

4.

\section{Summary}

The analysis of the data in Tables 1, 2 and of the distribution of density plotted in Fig. 3 drive to the following conclusions:

1) the density of galaxies, for square arcminute, in Seyfert environment exceeds of $30 \%$ the density in non active environment;

2)the distribution of density of galaxies in Seyfert environment as a function of D is a factor $1.3-1.4$ higher than in the environment of normal galaxies; 
3) a higher density of galaxies is found in both cases within the first diameter.

Further work will be devoted to check whether these trends are confirmed for galaxies belonging to different ranges of magnitude starting from the magnitude of the galaxy. In this way we will better check in first approximation whether the nearby environment of Seyfert galaxies is different from that of normal galaxies.

\section{References}

Barnes, J.E., \& Hernquist, L. 1991, Astrophys. J. 370, L65.

Dahari O., 1985, Astrophys. J. Suppl. 57, 643.

Davis M., Huchra J., Latham D., 1983, in: G. Abell \& G. Chincarini (eds.): IAU Symp. 104, Early Evolution of the Universe and Its Present Structure, Reidel, Dordrecht.

Fuentes Williams T., \& Stocke J. T., 1988, Astron. J. 96, 1235.

Gunn, J. 1979, in: C. Hazard and S. Mitton (eds.): Active Galactic Nuclei, Cambridge University Press, Cambridge, p. 213.

Huchra J., 1976, Astron. J, 81, 952.

Huchra J., Davis M., Latham D., Tonry J. 1983, Astrophys. J. Suppl. 52, 89.

Huchra J., Burg R. 1992, Astrophys. J. 393, 90.

Kogoshvili N., 1986, Merged Catalogue of Galaxies (unpublished, distributed with the Catalogue by the Centre des Données Stellaires).

Laurikainen E., \& Salo H., 1995, Astron. \& Astrophys. 293, 683.

MacKenty J. W., 1989, Astron. J. 343, 125.

Salvato M., 1996, Thesis

Rafanelli P., Violato M., \& Baruffolo A. 1995, Astron. J. 109, 1546.

Addresses of the authors:

M. Salvato, P. Rafanelli

Department of Astronomy

Vicolo dell'Osservatorio, 5

I-35122 Padova

Italy 